\title{
Influenza A virus-induced release of interleukin-10 inhibits the anti-microbial activities of invariant natural killer T cells during invasive pneumococcal superinfection
}

\author{
A Barthelemy ${ }^{1,2,3,4,5}$, S Ivanov ${ }^{1,2,3,4,5}$, J Fontaine $e^{1,2,3,4,5}$, D Soulard ${ }^{1,2,3,4,5}$, H Bouabe , C Paget $^{1,2,3,4,5}$, \\ C Faveeuw ${ }^{1,2,3,4,5}$ and F Trottein ${ }^{1,2,3,4,5}$
}

During influenza A virus (IAV) infection, changes in the lung's physical and immunological defenses predispose the host to bacterial superinfections. Invariant natural killer T (iNKT) cells are innate-like T lymphocytes that have beneficial or harmful functions during infection. We investigated the iNKT cells' role in a model of invasive pneumococcal superinfection. The use of $J \propto 18^{-/-}$mice indicated that $i N K T$ cells limited susceptibility to influenza-pneumococcal infection and reduced the lethal synergism. This role did not depend on immune-based anti-bacterial mechanisms. At the time of bacterial exposure, iNKT cells from IAV-experienced mice failed to produce antipneumococcal interferon- $\gamma$ and adoptive transfer of fresh iNKT cells before Streptococcus pneumoniae challenge did not restore anti-bacterial host defenses. Impaired iNKT cell activation in superinfected animals was related to the IAV-induced immunosuppressive cytokine interleukin-10 (IL-10), rather than to an intrinsic functional defect. IL-10 dampened the activation of iNKTcells in response to pneumococci by inhibiting the production of IL-12 by pulmonary monocyte-derived dendritic cells.

Neutralization of IL-10 restored iNKT cell activation and tends to increase resistance to secondary bacterial infection. Overall, iNKT cells have a beneficial role (upstream of bacterial colonization) in controlling influenza-pneumococcal superinfection, although they represent novel targets of immunosuppression at the time of bacterial challenge.

\section{INTRODUCTION}

Influenza A virus (IAV) infection can predispose the host to bacterial superinfections. The latter result in high morbidity and mortality rates during seasonal influenza $\mathrm{H} 1 \mathrm{~N} 1$ and $\mathrm{H} 3 \mathrm{~N} 2$ epidemics and pandemics (for reviews see Brundage ${ }^{1}$ and Morens et $a l^{2}{ }^{2}$. The encapsulated bacterium Streptococcus pneumoniae (a leading cause of mortality worldwide, comprising a group of more than 90 serotypes $^{3}$ ) is the predominant source of bacterial superinfections. Heightened susceptibility to bacterial superinfections is a multifactorial phenomenon that depends strongly on the pathogenicity index of IAV and on the host's immune status. ${ }^{2,4-6}$ Experiments in murine models suggest that disruption of the respiratory epithelium's barrier function, impairment of mucosal-ciliary clearance, and/or exposure of new bacterial attachment sites have a key role in bacterial adherence, colonization, and invasiveness., ${ }^{2,7}$ Furthermore, alteration of the innate immune response is a cardinal feature of postinfluenza bacterial superinfections. In particular, poor bacterial control is due to the loss and/or dysfunction of macrophages and neutrophils (e.g., the ability to sense and clear bacteria). ${ }^{10-14}$ Along with these effector cells, dysfunction of lymphoid cells, such as natural killer (NK) cells and $\gamma \delta \mathrm{T}$ lymphocytes, also depresses the host's anti-bacterial capabilities. ${ }^{15-17}$

Invariant NKT ( $i \mathrm{NKT}$ ) cells are lipid-reactive, non-conventional $\mathrm{T}$ lymphocytes that express an invariant $\mathrm{T}$-cell receptor (TCR)- $\alpha$ chain coupled to a small number of $\beta$-chains. ${ }^{18,19}$ In response to TCR triggering following antigen

${ }^{1}$ Université de Lille, U1019-UMR 8204—CIIL—Centre d'Infection et d'Immunité de Lille, Lille, France. ${ }^{2}$ CNRS, UMR 8204, Lille, France. ${ }^{3}$ Inserm, U1019, Lille, France. ${ }^{4} \mathrm{CHU}$ Lille, Lille, France. ${ }^{5}$ Institut Pasteur de Lille, Lille, France and ${ }^{6}$ Babraham Institute, Cambridge, UK. Correspondence: F Trottein (francois.trottein@pasteur-lille.fr) 
presentation by the CD1d molecule and/or in response to the stress-induced release of cytokines by accessory cells, $i \mathrm{NKT}$ cells rapidly produce large amounts of types 1, 2, and/or 17 cytokines. Conventional dendritic cells (DCs) are particularly potent $i$ NKT cell activators. In the context of infection, activation of Toll-like receptors expressed by DCs is a critical event in $i$ NKT cell activation. ${ }^{18,19}$ Cytokine production by $i \mathrm{NKT}$ cells is instrumental in trans-activating cells from the innate immune system (e.g., macrophages, neutrophils, and NK cells) and the adaptive immune system, which thus promote anti-infective effector responses. ${ }^{18,19}$ Invariant NKT cells are key players in pulmonary immunity. ${ }^{20}$ In particular, the cells actively participate in host defenses against respiratory viral and bacterial (including pneumococci) pathogens. ${ }^{21-26}$ In contrast, $i$ NKT cells can also exert harmful functions by exacerbating lung inflammation, as observed during asthma and chronic obstructive pulmonary disease for example. ${ }^{27,28}$

The potential function of $i$ NKT cells in postinfluenza bacterial superinfection has not previously been investigated. The events that precede postinfluenza bacterial colonization influence the host's susceptibility to secondary infections. We and others have emphasized the beneficial role of $i$ NKT cells in terms of anti-viral immunity and/or lung immunopathology during influenza $\mathrm{H} 1 \mathrm{~N} 1$ and $\mathrm{H} 3 \mathrm{~N} 2$ infections in animal models. ${ }^{29-32}$ The regulatory roles of $i \mathrm{NKT}$ cells are mediated through their impact on (i) the generation and expansion of IAV-specific $\mathrm{CD}^{+} \mathrm{T}$ lymphocytes and (ii) the controlled recruitment of myeloid-derived suppressor cells and inflammatory monocytes in the lungs. ${ }^{29,30,32}$ It has been suggested that the early production of interleukin-22 (IL-22) by $i$ NKT cells might control lung tissue damage and reinforce barrier functions during IAV infection ${ }^{31,33}$. Furthermore, IL-33 synthesis by $i$ NKT cells was suggested to activate group 2 innate lymphoid cells, ${ }^{34}$ a population that has beneficial functions on tissue repair during IAV infection. ${ }^{35}$ Given that the response to a single IAV infection is not predictive of the outcome of a superinfection, ${ }^{4,6}$ we decided to investigate the role of $i \mathrm{NKT}$ cells in postinfluenza invasive pneumococcal superinfection.

\section{RESULTS}

Invariant NKT cells limit the lethal synergy between IAV and pneumococcal infections

Wild-type (WT) mice and $i$ NKT-cell-deficient $J \alpha 18^{-/-}$mice were intranasally infected with H3N2 IAV. Seven days postinfection (dpi), the animals were inoculated with the invasive $S$. pneumoniae serotype 1 (Figure 1a). Low viral and bacterial inoculum levels were used to promote pathogen synergy (Supplementary Figure 1 online). Whereas the dose of S. pneumoniae was self-limiting as a single infection, IAV-experienced mice were susceptible to pneumococcal challenge as reflected by the presence of bacteria in the lungs and spleen, indicating systemic dissemination (Figure 1b). Interestingly, the absence of $i$ NKT cells was associated with greater bacterial loads in superinfected
$J \alpha 18^{-/-}$mice (relative to the WT), both within and outside the lungs.

We next sought to assess the impact of $i$ NKT cell deficiency on the lung's histopathological features. Lung samples from influenza-pneumococcus-infected WT mice displayed moderate-to-severe, multifocal, neutrophil-predominant pneumonia with interstitial lymphohistiocytic infiltration (Figure 1c). The histopathological features were more marked in superinfected $J \alpha 18^{-/-}$mice, which displayed very severe, fibrinopurulent, bronchial-interstitial pneumonia, large areas of consolidation, and blocked airways.

Mortality during pneumococcal superinfection is due to systemic bacterial dissemination and/or to excessive pneumonia. Both causes of mortality were accentuated in superinfected $J \alpha 18^{-/-}$mice (relative to the WT). Consistently, $\sim 70 \%$ of IAV-experienced WT mice died 2-5 days after pneumococcal infection, whereas all $J \alpha 18^{-/-}$mice died from bacterial superinfection (Figure 1d). Taken as a whole, our results show that a lack of $i$ NKT cells worsens pneumococcal superinfection and exacerbates disease.

\section{Influenza curtails INKT cell activation upon pneumococcal challenge}

We next looked at whether the positive impact of $i$ NKT cells during superinfection was due to indirect anti-bacterial effects. In the context of $S$. pneumoniae infection alone, pulmonary $i$ NKT cells produced interferon- $\gamma($ IFN- $\gamma$ ) and (to a lesser extent) IL-17A and the cells contributed to protection, as evidenced by a greater bacterial load in $J \alpha 18^{-/-}$mice, relative to WT animals (Figure 2a and Supplementary Figure 2A). The results of adoptive transfer experiments indicated that the production of IFN- $\gamma$ (but not IL-17A, data not shown) by $i$ NKT cells is important for controlling bacterial outgrowth and dissemination (Figure 2a and Supplementary Figure 2A, right panels).

In the context of IAV infection, pulmonary $i \mathrm{NKT}$ cells failed to produce IFN- $\gamma$ and IL-17A in response to $S$. pneumoniae (Figure 2b). Abrogation of cytokine production was not due to an intrinsic defect. Indeed, IAV-experienced $i$ NKT cells produced IFN- $\gamma$ and IL-17A in response to in vitro phorbol myristate acetate/ionomycin stimulation (Figure 2c). These findings suggested that $i \mathrm{NKT}$ cells are not exhausted and that cell-extrinsic factors contribute to the lack of cytokine production by $i$ NKT cells in superinfected animals. The use of neutralizing antibodies (Abs) indicated that IL-12, but not CD1d, is critical for the activation of pulmonary $i$ NKT cells (at least IFN- $\gamma$ ) in the context of $S$. pneumoniae infection alone (Supplementary Figure 2B). Interestingly, $i$ NKT cell dysfunction was associated with low transcript levels of Il12p40 and Il12p35 in the lungs of superinfected animals (Supplementary Figure 2C). Finally, as depicted in Figure 2d, adoptive transfer of fresh $i \mathrm{NKT}$ cells to IAV-experienced animals one day before the $S$. pneumoniae challenge did not restore anti-bacterial host defenses as reflected by bacterial counts. Collectively, IAV appears to induce an immunosuppressive environment in the lungs that 
a

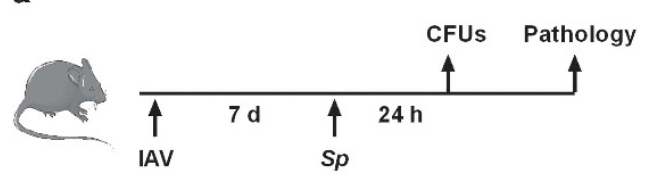

b

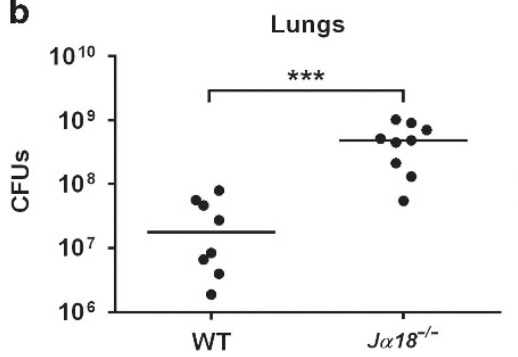

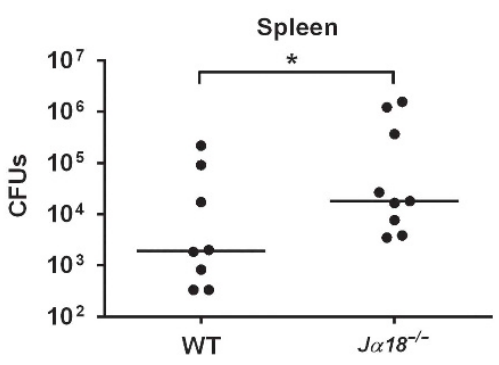

c
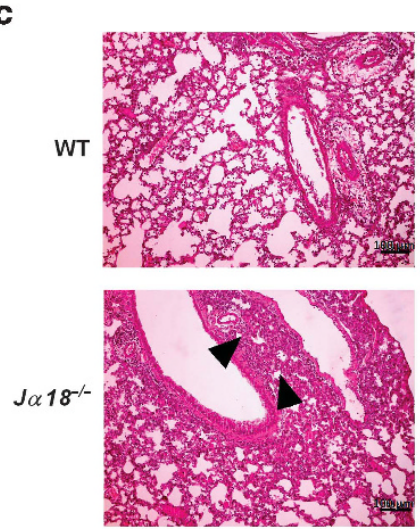

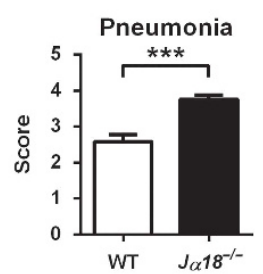

Distribution

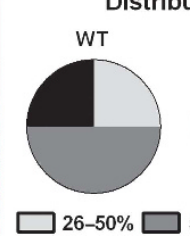

d

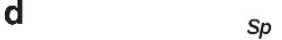

d $s p$

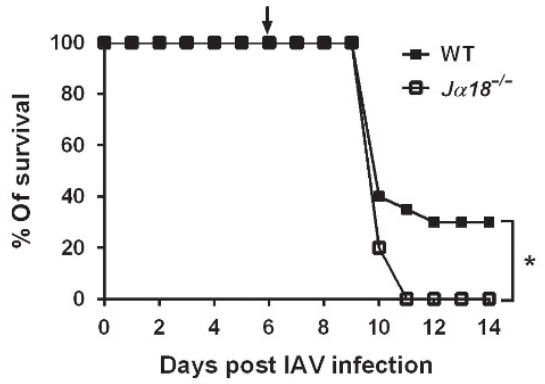

$51-75 \%-76-100 \%$

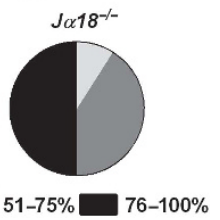

Figure 1 Role of invariant natural killer T (NKT) cells in influenza A virus (IAV)-pneumococcal infection. (a) An overview of the superinfection procedure. First, age-matched wild-type (WT) and $J \alpha 18^{-1-}$ male mice were intranasally infected with IAV (30 plaque-forming units). After 7 days, the mice were challenged with Streptococcus pneumoniae (Sp, $10^{3}$ colony-forming units (CFUs)). (b) The number of CFUs was determined in lungs and spleen $24 \mathrm{~h}$ after S. pneumoniae challenge. Each symbol represents one mouse; horizontal line indicates the median value. No bacteria were found in single-infected mice regardless of the mouse strain (data not shown). One of five representative experiments is shown. ${ }^{*} P<0.05$, ${ }^{* * \star} P<0.001$ (in a Mann-Whitney $t$-test). (c) Analysis of lung inflammation in superinfected WT and $J \alpha 18^{-1-}$ mice ( $36 \mathrm{~h}$ after $S$. pneumoniae challenge). Left panel: Representative hematoxylin plus eosin-stained tissue sections (original magnification $\times 100$ ). Arrows indicate lung consolidation. Right upper panel: Lung sections were scored blind for pneumonia. The data are reported as the mean \pm s.d. ( $n=12$ mice per group, combined from two independent experiments). ${ }^{* * *} P<0.001$ (in a Wilcoxon's test). Right lower panel: The surface distribution of lesions within the lungs are coded as follows: $26-50 \%$ (light gray), $51-75 \%$ (dark gray), and $>75 \%$ (black). (d) The survival of superinfected WT and $J \alpha 18^{-1-}$ mice was monitored ( $n=12-16$ mice per group, two pooled experiments). ${ }^{*} P<0.05$ (in a Kaplan-Meier analysis and log-rank test).

dampens $i$ NKT cell activation in the context of secondary pneumococcal infection.

\section{Influenza-induced IL-10 abrogates INKT cell activation (IFN- $\gamma$ ) upon pneumococcal challenge}

We next monitored the expression of immunosuppressive cytokines that might have accounted for the inhibition of $i$ NKT cell activation in superinfected animals. Influenza induced a high level of IL-10 expression in lungs (mRNA and protein), whereas the expression of TGF- $\beta(T g f b)$ and IL-35 (Il12p35/Ebi3) was unchanged at $7 \mathrm{dpi}$ (Figure 3a). Infection of IL-10- $\beta$-lactamase reporter mice identified $\mathrm{T}$ lymphocytes as a major source of IL-10 (Figure $3 \mathbf{b}$ and Supplementary Figure 3A). Furthermore, NK cells and myeloid cells (mostly inflammatory monocytes) expressed IL-10, whereas $i$ NKT cells did not (Supplementary Figure 3A,B and data not shown).

We next assessed potential contribution of IL-10 to the control of cytokine production by $i$ NKT cells in superinfected mice. In vivo blockade of IL-10 activity was sufficient to rescue IFN- $\gamma$ production by $i$ NKT cells but had no impact on IL-17A production (Figure $3 \mathbf{c}$ ). This effect was associated with enhanced levels of Il12p40 and Il12p35 (but not Il18, Il1b, and Il23p19) transcripts and bioactive IL-12 in the lung (Figure 3d). These results show that influenza-induced IL-10 is a critical factor in preventing the IL-12-driven activation of pulmonary iNKT cells (IFN- $\gamma$ ) upon pneumococcal challenge.

\section{IL-10 targets MoDCs to prevent NKT cell activation (IFN- $\gamma$ ) in superinfected mice}

IL-10 is known to suppress IL-12 production by accessory cells. Before seeking to identify the accessory cells targeted by IL-10, we first looked at whether IL-10 could act directly on $i$ NKT cells. Lung $i \mathrm{NKT}$ cells from IAV-infected mice did not express detectable levels of IL-10 receptor, as assessed by flow cytometry (Figure 4a, left panel). Furthermore, recombinant IL-10 had no effect on IFN- $\gamma$ and IL-17A production by lung $i$ NKT cells stimulated in vitro with IL-12p70/IL-18 (a cocktail that promotes IFN- $\gamma$ release under physiological conditions) or IL1- $\beta /$ IL-23 (for IL-17A), respectively (Figure 4a, right panel, and Supplementary Figure 4A).

Strikingly, our analysis of accessory cells in the lung of IAV-infected animals (7 dpi) revealed a very low number of conventional DCs (Figure $\mathbf{4 b}$ ), which are key activators of $i$ NKT cells during pneumococcal infections alone 


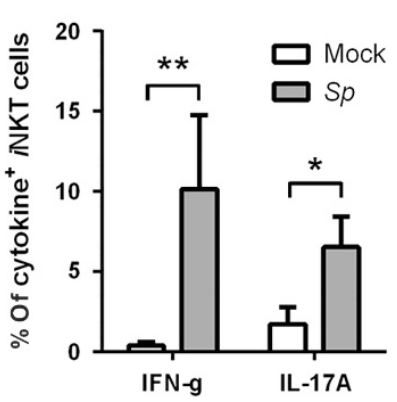

c

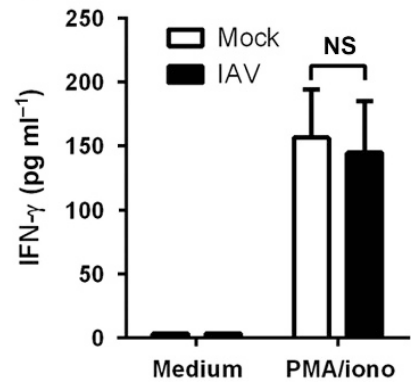

Sp only

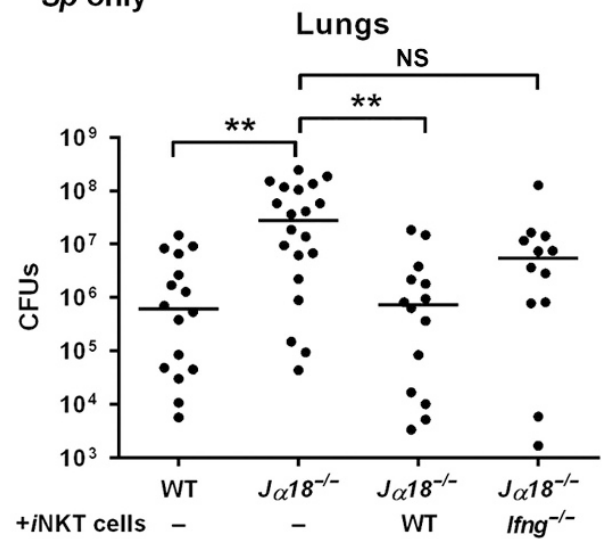

b

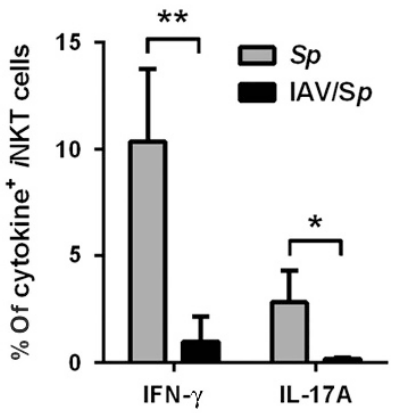

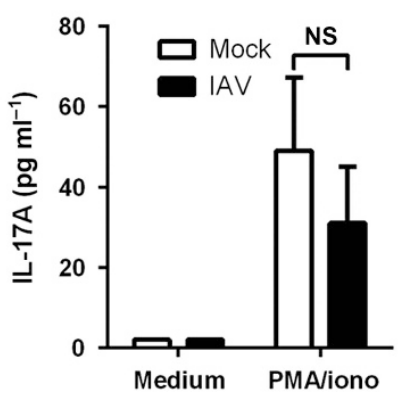

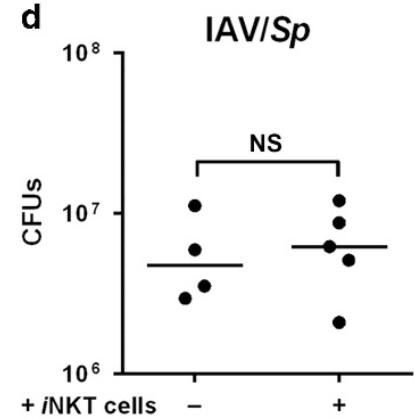

Figure 2 Role of invariant natural killer T (NKT) cells during pneumococcal infection and influenza-pneumococcal infection. (a) Left panel: Mice were infected with Streptococcus pneumoniae $\left(10^{6}\right.$ colony-forming units (CFUs)). Gated INKT cells (TCR- $\beta^{+}$PBS57-loaded CD1d tetramer $\left.{ }^{+}\right)$were analyzed for intracellular interferon- $\gamma$ (IFN- $\gamma$ ) and interleukin-17 (IL-17A) production $36 \mathrm{~h}$ after $S$. pneumoniae challenge. The mean percentages of pulmonary NKKT cells positive for IFN- $\gamma$ and IL-17A \pm s.d. is shown ( $n=8$, two pooled experiments). Right panel: $J \alpha 18^{-/-}$mice were reconstituted (or not) with WT or IFN$\gamma$-deficient NKT cells $24 \mathrm{~h}$ before infection. Wild-type (WT) and $J \alpha 18^{-/-}$mice were infected with S. pneumoniae (10 ${ }^{6} \mathrm{CFUs}$ ). The number of CFUs in lungs was determined $36 \mathrm{~h}$ after $\mathrm{S}$. pneumoniae challenge. The horizontal lines indicate the median values. (b) Mock- or influenza A virus (IAV)-infected mice ( $7 \mathrm{dpi}$ ) were intranasally infected with S. pneumoniae $\left(10^{6} \mathrm{CFUs}\right)$. The data correspond to pooled results from two independent experiments $(n=6-$ 8). (c) Pulmonary INKT cells were sorted from naive and IAV-infected mice ( $7 \mathrm{dpi}$ ) and were stimulated with phorbol myristate acetate/ionomycin ( $100 \mathrm{ng} /$ $1 \mu \mathrm{g} \mathrm{ml}^{-1}$ ). IFN- $\gamma$ and IL-17A production was measured by enzyme-linked immunosorbent assay (ELISA) after $24 \mathrm{~h}$. The results of two pooled experiments are shown (mean \pm s.d.). (d) IAV-infected mice were inoculated (or not) at day 6 postinfection with $\mathbb{N K T}$ cells $24 \mathrm{~h}$ before $S$. pneumoniae challenge. The number of bacteria was determined in the lungs $24 \mathrm{~h}$ later. The experiment shown is representative of two. ${ }^{\star} P<0.05$ and ${ }^{\star \star} P<0.01$ (a Mann-Whitney $t$-test or, in a, right panel, a Kruskal-Wallis one-way analysis of variance (ANOVA)). NS, nonsignificant.

(Supplementary Figure 4B). In contrast, a strong influx of monocyte-derived DCs (MoDCs) and inflammatory monocytes was observed at $7 \mathrm{dpi}$ (Figure $4 \mathbf{b}$ and Supplementary Figures 4C,D). We then established cocultures to test whether these accessory cells from IAV-infected mice could activate naive $i$ NKT cells. When exposed ex vivo to pneumococci, MoDCs (but not inflammatory monocytes) triggered IFN $-\gamma$ release by $i \mathrm{NKT}$ cells; in contrast, there was little or no IL-17A release (Figure 4c and data not shown). We next assessed whether IL-10 modulates the accessory activities of MoDCs to activate $i$ NKT cells. Flow cytometry experiments revealed that MoDCs expressed the IL-10 receptor (Figure 4d). Furthermore, in the above-mentioned coculture system, addition of exogenous IL-10 fully abrogated IFN- $\gamma$ production by $i$ NKT cells (Figure $4 c$ ). We conclude that MoDCs (but not inflammatory monocytes) from IAVinfected mice can activate $i$ NKT cells in the context of $S$. pneumoniae exposure, and that this phenomenon is inhibited by IL-10. To confirm this in vivo, IL-10 activity from IAV-infected mice was neutralized just before $S$. pneumoniae challenge (using the anti-IL-10 receptor $\mathrm{Ab}$ ) and sorted MoDCs were cocultured with naive $i \mathrm{NKT}$ cells. Compared with the control, MoDCs sorted from IL-10 receptor Ab-treated superinfected animals induced higher levels of IFN- $\gamma$ release by $i$ NKT cells (Figure 4e). This effect was associated with increased Il12p40 and Il12p35 (but not Il18) transcript expression by MoDCs (Supplementary Figure 4E and not shown).

\section{IL-10 neutralization tends to improve the outcome of influenza-pneumococcal superinfection}

We next investigated the functional consequences of IL-10 neutralization on bacterial superinfection. Wild-type IAV-infected mice treated with the anti-IL-10 receptor Ab just before the pneumococcal challenge tended to display a lower bacterial load than isotype-treated superinfected WT animals (Figure 5a). Furthermore, neutralization of IL-10 activity increased the survival rate of superinfected WT mice, albeit in a nonsignificant manner (Figure 5b). To investigate whether this partially protective effect was mediated by $i$ NKT cells, the same 

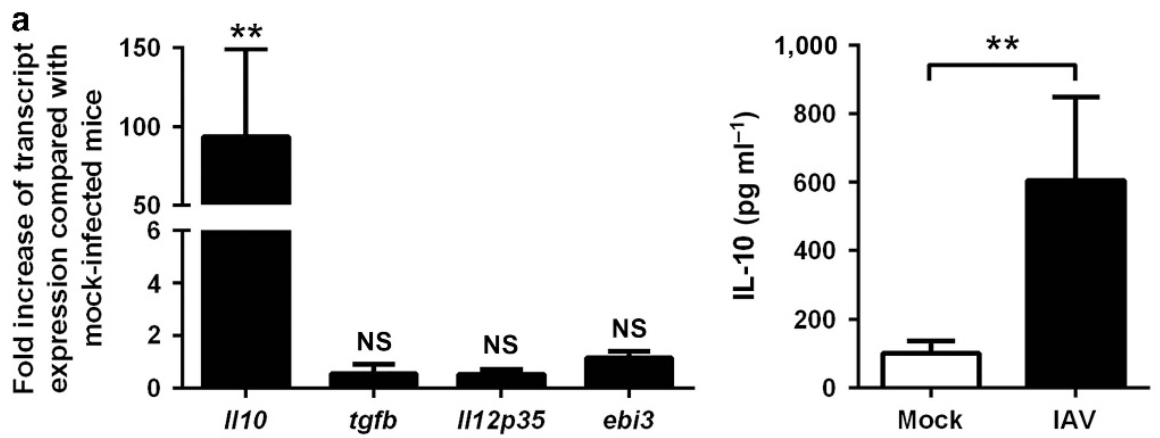

b
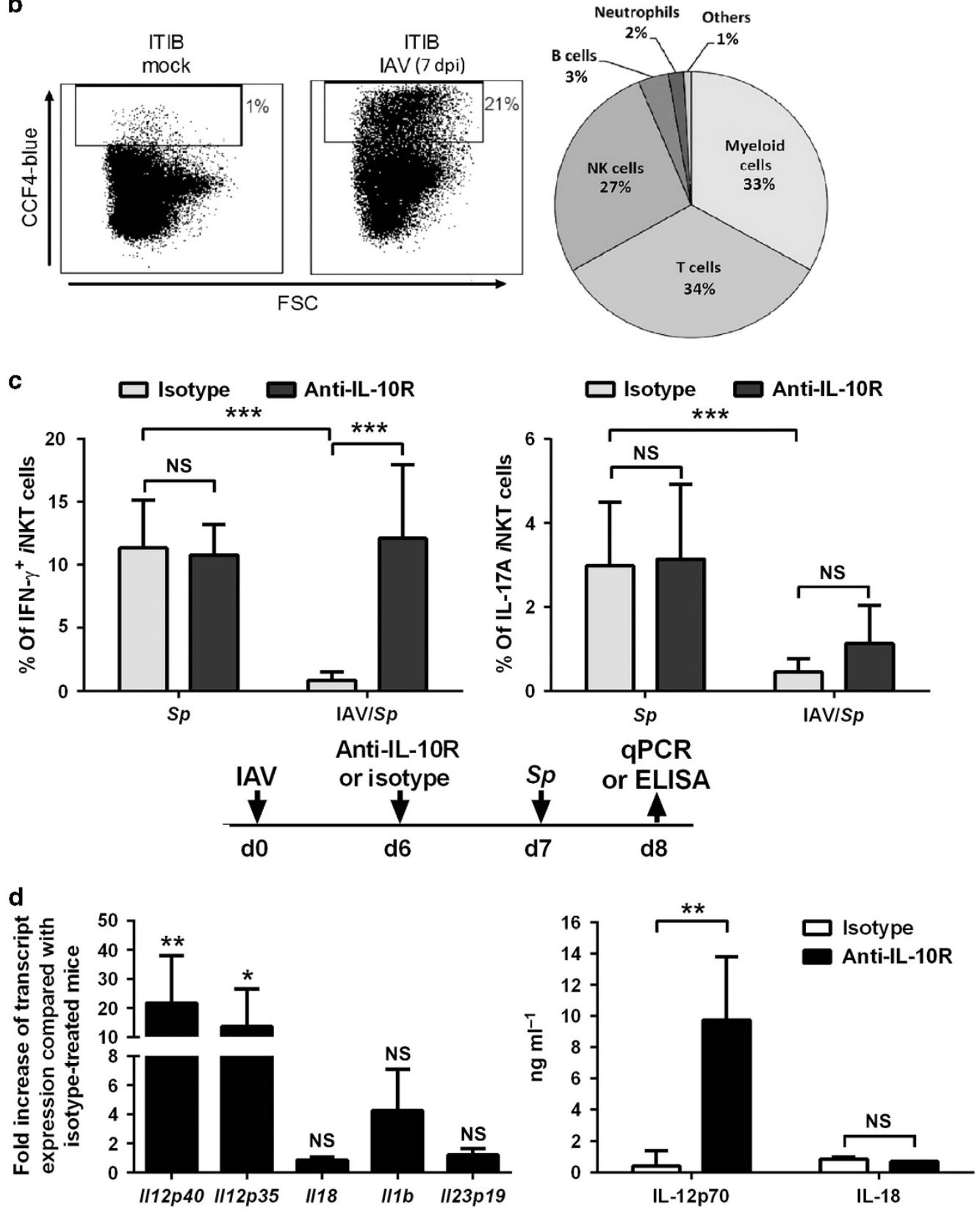
experiment was performed in $J \alpha 18^{-/-}$mice ( $i$ NKT cells cannot be depleted in WT animals). Treatment with the antiIL10 receptor $A b$ reduced the bacterial load in superinfected $J \alpha 18^{-/-}$mice (Figure 5a). However, this reduction in the bacterial load (a key parameter in the outcome of bacterial superinfection) was not as intense as in WT mice. Last, neutralizing IL-10 increased the survival rate in superinfected $J \alpha 18^{-/-}$mice, although the degree of protection was lower relative to WT animals. We conclude that (i) IL-10 neutralization tends to improve the outcome of an IAV-pneumococcal infection and (ii) this effect might depend partly on $i \mathrm{NKT}$ cells.

\section{DISCUSSION}

Although murine $i$ NKT cells clearly exert a positive role in primary infections by serotype 3 pneumococci ${ }^{23-26}$ and the a

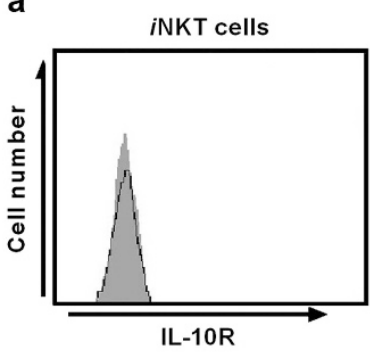

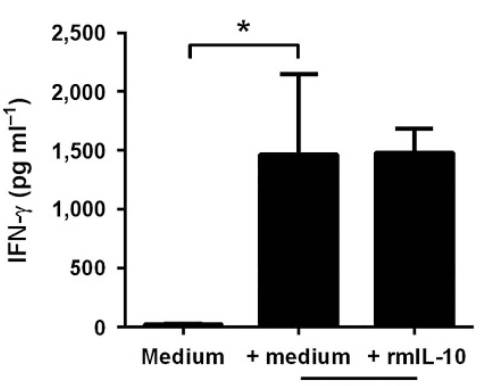

$\overline{\mathrm{IL}-12 / \mathrm{IL}-18}$

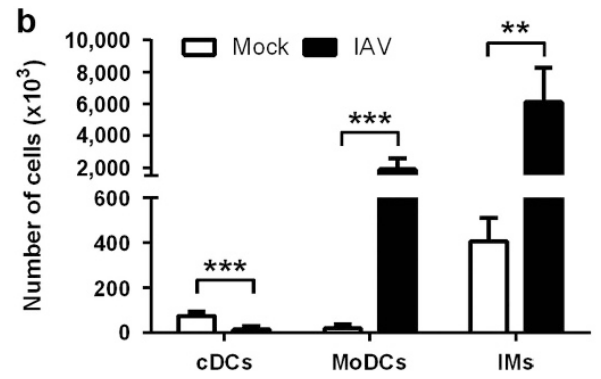

C

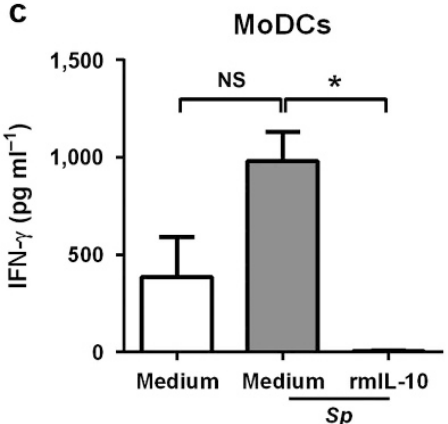

IMs

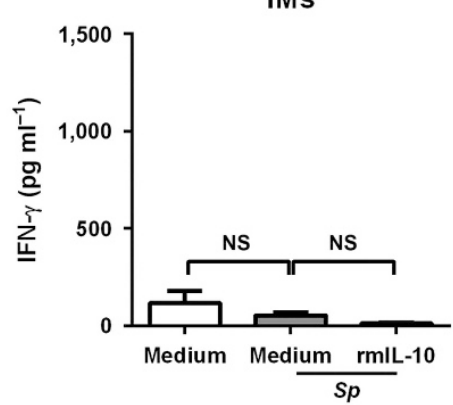

d

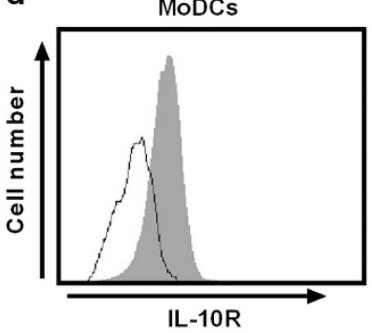

e

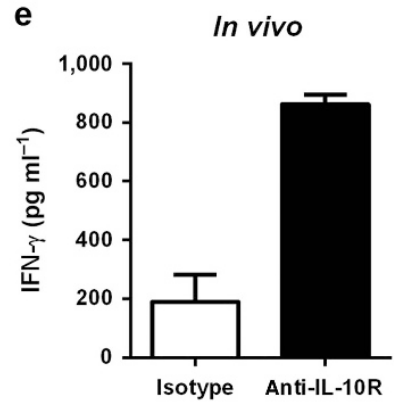

Figure 4 Mode of action of interleukin-10 (IL-10) in the inhibition of invariant natural killer T (NKT) cell activation during coinfection. (a) Left panel: The expression of the IL-10 receptor on NKT cells (7 dpi). White histograms: Isotype control; gray histograms: IL-10 receptor. Right panel: Lung 1 NKT cells from influenza A virus (IAV)-infected mice were stimulated in an antigen-presenting cell-free system with IL-12p40 plus IL-18 in the presence or absence of recombinant mouse IL-10 (10 $\left.\mathrm{ng} \mathrm{ml}^{-1}\right)$. The concentration of interferon- $\gamma(\mathrm{IFN}-\gamma)$ (duplicates $\pm \mathrm{s}$. $\mathrm{d}$.) in the supernatant was determined after $24 \mathrm{~h}$. Two experiments were pooled. (b) The mean \pm s.d. proportions of conventional dendritic cells (DCs), monocyte-derived DCs (MoDCs), and inflammatory monocytes (IMs) among CD45 ${ }^{+}$cells were determined in mock- and IAV-infected ( $\left.7 \mathrm{dpi}\right)$ mice $(n=8$, two pooled experiments). (c) MoDCs and IMs were sorted from IAV-infected mice, incubated with Streptococcus pneumoniae (at a multiplicity of infection of 1 ) for $1 \mathrm{~h}$, washed, and then cocultured with naive pulmonary INKT cells in the absence or presence of IL-10 $\left(10 \mathrm{ng} \mathrm{ml}^{-1}\right)$. The concentration of IFN- $\gamma$ (mean \pm s.d., triplicates) was determined after $48 \mathrm{~h}$. One of two representative experiments is shown. (d) The expression of the IL-10 receptor on MoDCs (7 dpi). White histograms: Isotype control; gray histograms: IL-10 receptor. (e) IAV-infected mice were treated with the anti-IL-10 receptor or the isotype control monoclonal antibody (mAb) $24 \mathrm{~h}$ before $S$. pneumoniae challenge. In vivo S. pneumoniae-exposed MoDCs were then sorted ( $24 \mathrm{~h}$ after the pneumococcal challenge) and incubated with naive pulmonary $\mathbb{N K T}$ cells. The mean \pm s.d. concentration of IFN- $\gamma$ (in duplicate) is shown. One of two representative experiments is shown. ${ }^{*} P<0.05$, ${ }^{\star *} P<0.01$, and ${ }^{\star * *} P<0.001$ (in a Mann-Whitney $t$-test in $\mathbf{b}$ or a Kruskal-Wallis one-way analysis of variance (ANOVA) in a and $\mathbf{c}$ ).

Figure 3 Cell sources of interleukin-10 (IL-10) during primary influenza, and the role of IL-10 in the inhibition of invariant natural killer T (NKT) cell activation in superinfected mice. (a) Left panel: RNA was extracted from the lungs of mock-infected and influenza A virus (IAV)-infected mice (7 dpi). I/10, Tgfb, I/12p35, and Epstein-Barr virus-induced gene 3 (Eb/3) mRNA copy numbers were measured by quantitative reverse transcription-PCR (RT-PCR). Data are expressed as the mean \pm s.d. fold increase over average gene expression in mock-treated mice $(n=6)$. Right panel: IL-10 production was measured in bronchoalveolar lavage fluids at $7 \mathrm{dpi}$. One of two representative experiments is shown $(n=5)$. (b) Left panel: A representative dot plot of $\mathrm{CD}_{4}{ }^{+}$propidium iodide (PI) ${ }^{-}$cells expressing CCF4-blue fluorescence (corresponding to IL-10- $\beta$-lactamase ${ }^{+}$cells). Right panel: The percentage of

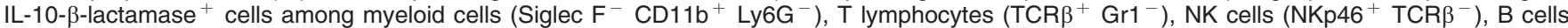
$\left(\mathrm{Ly}_{6 \mathrm{G}}{ }^{-} \mathrm{CD}_{19}{ }^{+}\right)$, and neutrophils $\left(\mathrm{CD} 11 \mathrm{~b}^{+} \mathrm{Ly}_{6 \mathrm{G}}{ }^{+}\right.$Siglec $\left.\mathrm{F}^{-}\right)(n=12$, three independent experiments). (c) IAV-infected mice were injected with the anti-IL-10 receptor (IL-10R) or the isotype control mAb (1 mg) $24 \mathrm{~h}$ before Streptococcus pneumoniae challenge. For each group, the percentage $\pm \mathrm{s}$. $\mathrm{d}$. of IFN- $\gamma$ - and IL-17A-producing NKT cells is shown $(n=6-12$, three pooled experiments). (d) The same experiment as in (c) but with killing $24 \mathrm{~h}$ after S. pneumoniae infection ( $36 \mathrm{~h}$ in c). Left panel: Data are expressed as the mean $\pm \mathrm{s}$.d. fold increase over average gene expression in isotype-treated superinfected mice $(n=6)$. Right panel: The mean concentrations \pm s.d. of bioactive IL-12 and IL-18 (whole lung homogenates) are represented $(n=5)$. One of two representative experiments is shown. ${ }^{\star} P<0.05$, ${ }^{* *} P<0.01$, and ${ }^{* * *} P<0.001$ (Mann-Whitney $t$-test or, in $\mathbf{c}$, a Kruskal-Wallis one-way analysis of variance (ANOVA)). ELISA, enzyme-linked immunosorbent assay; NS, nonsignificant. 

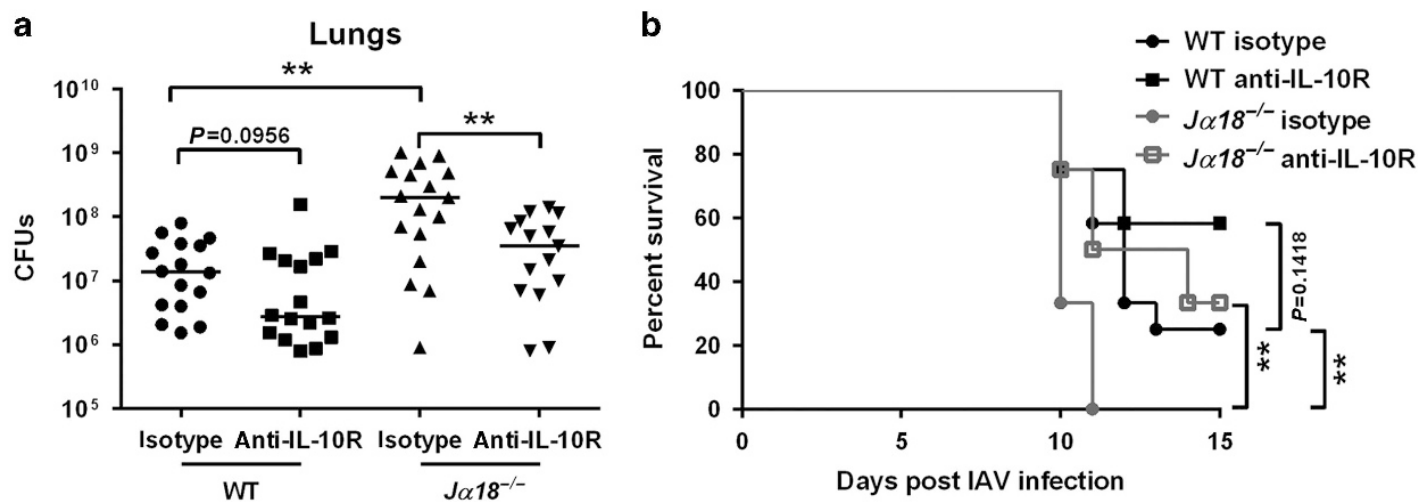

Figure 5 Effect of interleukin-10 (IL-10) neutralization in superinfected wild-type (WT) and Ja18 $18^{-/-}$mice. Influenza A virus (IAV)-infected WT and $J \propto 18^{-1-}$ mice were injected with the anti-IL-10 receptor (IL-10R) or the isotype control mAb (1 mg per mouse) $24 \mathrm{~h}$ before Streptococcus pneumoniae challenge. (a) Bacteria in the lungs were counted $24 \mathrm{~h}$ later (two pooled experiments). The horizontal lines indicate the median values $\left({ }^{\star *} P<0.01\right)$ (a Kruskal-Wallis one-way analysis of variance (ANOVA)). (b) The survival of superinfected mice was measured $(n=14-16$ mice per group, for two pooled experiments) and analyzed according to the Kaplan-Meier method and a log-rank test $\left({ }^{\star \star} P<0.01\right)$.

serotype 1 studied here (serotypes that differ in virulence, pathogenicity, and invasiveness ${ }^{3}$ ), the potential role of $i \mathrm{NKT}$ cells in pneumococcal superinfections following IAV infection has yet to be characterized. In view of their role (under some circumstances) in immune suppression and pulmonary immunopathology, ${ }^{18,19,27,28,36}$ a harmful role of $i$ NKT cells in the lethal synergy between IAV and pneumococci could not be ruled out. In the present study, we found that $i$ NKT cells have neither a detrimental, immunosuppressive role nor a harmful, proinflammatory function in influenza-pneumococcal superinfected mice. In fact, $i$ NKT cells reduce susceptibility to secondary pneumococcal infection and limit the lethal synergy between IAV and pneumococci.

During IAV infection, events that precede bacterial colonization impact on susceptibility to secondary infection. We and others have shown that $i$ NKT cells protect against lung damage and alteration of barrier functions during influenza. ${ }^{29-32}$ These previous observations and our present demonstration that (i) $i \mathrm{NKT}$ cells were not activated in mice infected with IAV and pneumococci (at least in terms of IFN- $\gamma$ and IL-17A production) and that (ii) in vivo transfer of naive $i$ NKT cells to IAV-infected mice failed to restore protection against pneumococci suggest that the beneficial action of $i \mathrm{NKT}$ cells on superinfection is exerted upstream of bacterial colonization and might be related to better physical defenses (rather than better immune-based defenses). Further studies are needed to accurately characterize the role of $i$ NKT cells in physical defenses against bacterial superinfections. This role might be exerted through a variety of nonredundant mechanisms. By controlling the recruitment of epithelial-damaging inflammatory monocytes during primary influenza, ${ }^{9,32} i \mathrm{NKT}$ cells might indirectly limit bacterial superinfection. One can also hypothesize that the early synthesis of the epitheliumprotecting cytokine IL- 22 by $i$ NKT cells ${ }^{31,33}$ might reinforce the respiratory barrier, ${ }^{31,37,38}$ thus limiting secondary infections. It is noteworthy that $I l 22^{-1-}$ mice displayed better outcomes following bacterial superinfection than WT counterparts. ${ }^{33}$
Last, through their ability to indirectly activate the synthesis of protective barrier factors by other cells (e.g., amphiregulin by group 2 innate lymphoid cells, ${ }^{34,35}$ ) $i$ NKT cells might lower epithelial damage during influenza and thus limit bacterial superinfection.

Depending on the bacterial dose and serotype, IFN- $\gamma$ and/or IL-17A have important functions in pneumococcal clearance. ${ }^{3,22}$ Our results demonstrate that following IAV infection, pulmonary $i \mathrm{NKT}$ cells become refractory to subsequent in vivo stimulation with $S$. pneumoniae-suggesting that the primary IAV infection inhibits the $i$ NKT cells' indirect bactericidal activities. This finding is in line with the fact that influenza cripples the functions of innate sensors (including Toll-like receptors) and compromises innate immunity. ${ }^{39}$ Further ex vivo analysis demonstrated that (i) $i \mathrm{NKT}$ cells conserved their ability to respond to inflammatory cytokines released by accessory cells and (ii) the lack of $i \mathrm{NKT}$ cell activation in superinfected animals was independent of changes in lung DC populations. Our results point toward a dominant role for IL-10 in curbing $i \mathrm{NKT}$ cell activation (at least IFN- $\gamma$ production) in superinfected mice, due to low IL-12 production. Virus-specific $\mathrm{T}$ cells were shown to be the main producers of IL-10 during IAV H1N1 infection. ${ }^{40,41}$ Our data extend these findings and provide evidence that, along with T lymphocytes, NK cells and myeloid cells (and especially inflammatory monocytes) contribute to IL-10 secretion during H3N2 influenza infection.

The modes of action of IL-10 on $i$ NKT cell functions are unclear, although a recent study showed that this cytokine induces human $i$ NKT cells to become tolerogenic and anergic. $^{42}$ In our experimental system, IL-10 did not directly target pulmonary $i$ NKT cells but did affect accessory cells. At $7 \mathrm{dpi}$, MoDCs constituted a major population of accessory cells in the lungs; these cells reportedly interact with newly recruited effector $\mathrm{CD}^{+}{ }^{+} \mathrm{T}$ lymphocytes and are involved in virus clearance. ${ }^{43}$ We found that MoDCs sensitized both ex vivo and (more importantly) in vivo with $S$. pneumoniae had the potential to activate $i$ NKT cells. This effect was inhibited by 
IL-10. MoDCs were not significant IL-10 producers (data not shown) and were themselves targets of IL-10-producing cells. It remains to be seen whether IL-10 produced by T cells, NK cells, and/or inflammatory monocytes prompts MoDCs to inhibit the activation of $i$ NKT cells in the context of superinfection.

The lack of IFN- $\gamma$ and/or IL-17A production by $i$ NKT cells in influenza-pneumococcal-infected mice might be of relevance. Indeed, Sun et al. ${ }^{11}$ suggested that excessive IFN- $\gamma$ production (by targeting macrophages) might be harmful in bacterial superinfections. In contrast, reduction of IFN- $\gamma$-producing IAV-specific $T$ cells in the lungs of IAV-pneumococcus contributes to postinfluenza superinfection. ${ }^{44}$ Last, in a model of IAV-Staphylococcus aureus infection, Kudva et al. ${ }^{45}$ showed that IFN- $\gamma$ has no role in bacterial outgrowth in superinfected animals. Therefore, the role of IFN- $\gamma$ in IAV bacterial infection might depend on the model used, and one can infer that IL-10-mediated inhibition of IFN- $\gamma$ production by $i$ NKT cells might be of relevance in bacterial superinfections. Several studies have demonstrated that defective synthesis of IL-17A (e.g., by $\gamma \delta \mathrm{T}$ cells) is associated with enhanced susceptibility to respiratory bacterial infections after influenza. ${ }^{16,17,45,46}$ Consistently, $i$ NKT cells failed to produce IL-17A in superinfected mice, a mechanism that might contribute to enhanced susceptibility to secondary pneumococcal infection.

By using a model of mild H1N1 IAV infection, Van der Sluijs et al. ${ }^{14,47}$ showed that neutralization of IL-10 (14 dpi) reduced the pulmonary outgrowth of $S$. pneumoniae (serotype 3 ) and improved survival during secondary bacterial pneumonia. The researchers attributed this positive effect to the enhancement of anti-bacterial host defenses. In line with these data, we found that neutralization of IL-10 during the acute phase of H3N2 influenza $(7 \mathrm{dpi}$ ) tended to reduce the bacterial load and improve the survival rate. Hence, IL-10 neutralization is associated with (i) restoration of $i$ NKT cell activation (at least IFN- $\gamma$ ) in superinfected animals and (ii) improved resistance to bacterial superinfection. In this setting, cytokines and/or chemokines released by $i \mathrm{NKT}$ cells might activate macrophages and/or neutrophils, which are important controllers of pneumococci outgrowth. ${ }^{3}$ It is noteworthy that a partially protective effect of IL-10 blockade was also observed in superinfected $J \propto 18^{-/-}$mice, indicating that IL-10 has an $i$ NKT-cell-independent action. In this setting, IL-10 might negatively act on other upstream innate immune cells and/or directly on macrophages and/or neutrophils. ${ }^{14}$

Taken as a whole, our present findings emphasize that $i$ NKT cells are early beneficial regulators of bacterial superinfection and represent previously unrecognized targets of immunosuppression during influenza. Our results provide insight into indirect IL-10-mediated inhibition of $i$ NKT cell activation in a context of IAV-pneumococcal superinfection. To control postinfluenza bacterial superinfections, future therapeutic approaches could seek to limit pulmonary damage upstream of bacterial colonization and/or overcome the immunosuppression. In the clinic, exploitation of $i$ NKT cells might therefore be of great value in reducing disease morbidity and mortality.

\section{METHODS}

Ethics statement. All animal work conformed with the Lille Pasteur Institute's regulations on animal care and use guidelines and was approved by the regional investigational review board (Comité d'Ethique en Expérimentation Animale Nord Pas-de-Calais: reference AF 16/20090) and the French Ministry for Research (Ministère de l'Education nationale, de l'Enseignement Supérieur et de la Recherche: references CEAA75 and 00357.03).

Reagents and Abs. Recombinant mouse IL-10, IL-12p70, IL-18, IL-1 $\beta$, and IL-23 were purchased from Peprotech (London, UK), CCF4-AM ( $\beta$-lactamase substrate), probenecid, and Live/Dead Cell Viability Kit were from Life Technologies (Saint Aubin, France), and diphtheria toxin was from Sigma (Saint-Quentin Fallavier, France). Monoclonal Abs (mAbs) against mouse TCR- $\beta$ (FITC, APC, or V450-conjugated), Ly6G (APCCy7 or FITC), Gr1 (AlexaFluor700 or PE), CD19 (APC), CD11c (PECy7), CD64 (APC), Siglec F (PacificBlue or PE), NKp46 (PECy7), CD45 (FITC, APCCy7, AlexaFluor700 or PE), MHC class II (Pacific-blue, BV605 or AlexaFluor700), CD11b (Percp-Cy5.5), Ly6C (AlexaFluor700), IL-10 receptor (PE), IFN- $\gamma$ (AlexaFluor-647 or PE), IL-17A (PE), and isotype controls were purchased from BD Pharmingen (Le Pont de Claix, France). The aqua fluorescent reactive dye (LIVE/DEAD) was purchased from Life Technologies. Phycoerythrin-conjugated PBS-57- (a glycolipid analog of $\alpha$-galactosylceramide) loaded CD1d tetramer was from the NIAID Tetramer Facility (Emory University, Atlanta, GA). Neutralizing mAbs against IL-12p40 (C17.8), CD1d (19G11), IL-10 receptor (1B1.3A), and isotype control mAbs (2A3, LTF-2, HRPN) were from Bio X Cell (West Lebanon, NH).

Animals. Eight-week-old male C57BL/6 mice were purchased from Janvier (Le Genest-St-Isle, France). CD11c-DTR and J $\alpha 18^{-1-}$ mice have been described previously. ${ }^{48,49} \mathrm{~J} \alpha 18^{-1-}$ mice are completely devoid of $i$ NKT cells. Ifng ${ }^{-/-}$mice were obtained from the Jackson Laboratory (Bar Harbor, ME). For IAV and S. pneumoniae infection, mice were maintained in a biosafety level 2 facility in the Animal Resource Center at the Lille Pasteur Institute (Lille, France). To identify the cells that produced IL-10 during influenza, we took advantage of the highly sensitive IL-10- $\beta$-lactamase reporter mouse (the ITIB mouse)..$^{50}$

Infection with IAV and/or S. pneumoniae. For infection with IAV alone, $50 \mu \mathrm{l}$ of phosphate-buffered saline (PBS) containing (or not, in a mock sample) 30 plaque-forming units of the high-pathogenicity, mouse-adapted H3N2 IAV strain Scotland/20/74 were intranasally administered to anesthetized mice. For infection with $S$. pneumoniae serotype 1 (clinical isolate E1586) alone, $50 \mu \mathrm{l}$ of a PBS suspension of bacteria $\left(10^{6}\right.$ colony-forming units, CFUs) were intranasally administered. Superinfection was performed as follows. Mice infected with IAV (30 plaque-forming units) 7 days earlier were intranasally inoculated with $10^{3} \mathrm{CFUs}$ of $S$. pneumoniae serotype 1 . In some cases, IAV-infected mice were inoculated with $10^{6} \mathrm{CFUs}$. The singly (S. pneumoniae) or doubly (IAV/S. pneumoniae) infected mice were monitored daily for mortality. Mice that were moribund (i.e. alive but lacking purposeful movement after stimulation or having lost over $20 \%$ of their initial weight) were killed and considered to have died on that day. Viable bacteria in the lungs and spleen were counted $36 \mathrm{~h}$ (single infection) or $24 \mathrm{~h}$ (coinfection) after the S. pneumoniae challenge by plating serial 10 -fold dilutions of lung or spleen homogenates onto blood agar plates. The plates were incubated at $37^{\circ} \mathrm{C}$ overnight and CFUs were counted $24 \mathrm{~h}$ later.

Histological analysis. For histopathologic assessment, lungs were fixed by inflation and immersion in $3.2 \%$ paraformaldehyde in PBS and embedded in paraffin. To evaluate airway inflammation, we stained the paraffin-embedded fixed lung slices (thickness: $5 \mu \mathrm{m}$ ) with hematoxylin-eosin reagent. Lung sections were scored by an experienced veterinary pathologist who was blinded to the 
composition of the groups. Bronchointerstitial pneumonia was scored on a scale of $1-5$, as described elsewhere. ${ }^{30}$

Assessment of gene expression by quantitative reverse transcription-PCR. Total RNA from whole lungs or from sorted pulmonary cells was extracted. The cDNAs were analyzed using quantitative reverse transcription-PCRs, as described elsewhere. ${ }^{33}$ Specific primers for $g a p d h, 5^{\prime}$-TGCCCAGAACATCATCCCTG- ${ }^{\prime}$ and $5^{\prime}$-TCAGATCCACGACGGACACA- $3^{\prime} ; i l 1 b, 5^{\prime}$-TCCCCAACT GGTACATCAGCA- ${ }^{\prime}$ and $5^{\prime}$-ACACGGATTCCATGGTGAAGT- ${ }^{\prime}$; $i l 10,5^{\prime}$-CATTTGAATTCCCTGGGTGAGA- $3^{\prime}$ and $5^{\prime}$-TGCTCCACT GCCTTGCTCTT-3'; il12p40, 5'-GACCCTGCCCATTGAACTGG C- $3^{\prime}$ and $5^{\prime}$-CAACGTTGCATCCTAGGATCG- $3^{\prime}$, il12p35, 5' ${ }^{\prime}$-CACG TCACCTCCTCTTTTTG- $3^{\prime}$ and $5^{\prime}$-CAGCAGTGCAGGAATAATG TT-3'; Il18, 5'-ATCAGACAACTTTGGCCGACT- $3^{\prime}$ and $5^{\prime}$-TCATATCCTCGAACACAGGCTG - $3^{\prime}$; Il23p19, $5^{\prime}$-AATCTCTGCATGC TAGCCTGG- $3^{\prime}$ and $5^{\prime}$-GATTCATATGTCCCGCTGGTG- $3^{\prime}$; tgf $b$, $5^{\prime}$-TGACGTCACTGGAGTTGTACGG- ${ }^{\prime}$ and $5^{\prime}$-GGTTCATGTCA TGGATGGTGC-3'; ebi3, 5'-CAATGCCATGCTTCTCGGTATC-3' and $5^{\prime}$-GCCTGTAAGTGGCAATGAAGGA- ${ }^{\prime}$ were designed using the Primer Express software (Applied Biosystems, Villebon sur Yvette, France). Data were normalized against expression of the gapdh gene and are expressed as a fold-increase over the mean gene expression level in mock-infected mice or isotype-treated superinfected animals. ${ }^{30}$

Analysis of iNKT activation and purification, adoptive transfer, and culture. Fixed cells were permeabilized and incubated with conjugated $\mathrm{mAbs}$ against IFN- $\gamma$, IL-17A, or control rat IgG1 mAb. The data were acquired on an LSRFortessa cytometer (Becton Dickinson Biosciences, Rungis, France) running FACSDiva software and were then analyzed with the FlowJo software. To study the mechanisms of $i$ NKT activation, mice were treated intraperitoneally with anti-IL-12p40 $(200 \mu \mathrm{g}$ per mouse), CD1d (500 $\mu$ g per mouse), or isotype controls $24 \mathrm{~h}$ before S. pneumoniae challenge. To deplete conventional DCs, mice that were heterozygous for the CD11c-DTR transgene were injected intraperitoneally with $100 \mathrm{ng}$ diphtheria toxin, as described elsewhere. ${ }^{49}$ $i$ NKT cells were purified by labeling mononuclear cells from the lung or liver with phycoerythrin-conjugated, PBS-57-loaded CD1d tetramer and an FITC-conjugated anti-TCR $\beta$ Ab. After cell surface labeling, the cells were sorted using a FACSAria cytometer (BD Biosciences, Rungis, France). The purity of PBS57-loaded CD1d tetramer ${ }^{+} \mathrm{TCR}^{+}$cells after sorting was consistently $>98 \%$. For cell culture experiments, pulmonary $i$ NKT cells $\left(5 \times 10^{3}\right.$ cells per well $)$ from mock- or IAV-infected mice $(7 \mathrm{dpi})$ were stimulated with phorbol myristate acetate $\left(100 \mathrm{ng} \mathrm{ml}^{-1}\right)$ plus ionomycin $\left(1 \mu \mathrm{g} \mathrm{ml}^{-1}\right)$, IL-12 $\left(50 \mathrm{ng} \mathrm{ml}^{-1}\right)$ plus IL-18 $\left(1 \mathrm{ng} \mathrm{ml}^{-1}\right)$, or IL-23 $\left(1 \mathrm{ng} \mathrm{ml}^{-1}\right)$ plus IL-1 $\beta\left(1 \mathrm{ng} \mathrm{ml}{ }^{-1}\right)$. IFN- $\gamma$ and IL-17A production was quantified in an enzyme-linked immunosorbent assay $24 \mathrm{~h}$ later. For reconstitution experiments, recipient $J \alpha 18^{-1-}$ mice were inoculated intravenously with $10^{6}$ liver $i$ NKT cells $24 \mathrm{~h}$ before the pneumococcal challenge.

\section{Analysis of IL-10- $\beta$-lactamase expression in pulmonary cells following IAV infection. Lung mononuclear cells from naive or IAV-infected ITIB mice and from a naive $\mathrm{C} 57 \mathrm{Bl} / 6$ mouse were pelleted, resuspended ( $10^{7}$ cells per $\mathrm{ml}$ ) in PBS containing CCF4-AM substrate $(1 \mu \mathrm{M})$ supplemented with probenecid $(2.5 \mathrm{mM})$, and then incubated in the dark for $45 \mathrm{~min}$ at room temperature. ${ }^{50}$ The cells were then washed two times with PBS/2\% fetal calf serum, labeled with appropriate Abs (for cell identification) and propidium iodide (to exclude dead cells) and analyzed by flow cytometry.}

Analysis of pulmonary conventional DCs, MoDCs, and inflammatory monocytes. Lung mononuclear cells from naive or IAV-infected mice were initially labeled for dead cells with the Live/Dead Cell Viability Kit according to the manufacturer's protocol. To identify conventional DCs and MoDCs, lung mononuclear cells were labeled with appropriate dilutions of FITC-conjugated anti-CD45, Brilliant-Violet 421-conjugated anti-Siglec-F, APC (allophycocyanin)-H7-conjugated anti-Ly6G, PE-Cy7-conjugated anti-CD11c, AlexaFluor 700-conjugated anti-MHC Class II, and APC-conjugated anti-CD64 Abs. Although cell types are CD45 ${ }^{+}$ Siglec-F ${ }^{-}$Ly6G $^{-} \mathrm{CD}_{11 \mathrm{c}^{+}}$and $\mathrm{MHC} \mathrm{II}^{+}$, conventional DCs are CD64-negative and MoDCs are CD64-positive. Inflammatory monocytes were identified as $\mathrm{CD}_{4}{ }^{+}$Siglec-F ${ }^{-} \mathrm{Ly} 6 \mathrm{G}^{-} \mathrm{Ly}^{+} \mathrm{C}^{+}$ $\mathrm{CD}_{11 \mathrm{~b}}{ }^{+} \mathrm{CCR}^{+}{ }^{+}$cells.

Purification of pulmonary MoDCs and inflammatory monocytes, and coculture experiments. MoDCs and inflammatory monocytes sorted from IAV-infected mice were exposed for $1 \mathrm{~h}$ to $S$. pneumoniae (at a multiplicity of infection of 1), washed two times with PBS containing antibiotics, and then cocultured with cell-sorted naive pulmonary $i$ NKT cells $\left(2 \times 10^{4}\right.$ accessory cells $/ 5 \times 10^{3} i \mathrm{NKT}$ cells per well) in the presence or absence of recombinant IL-10 $\left(1 \mathrm{ng} \mathrm{ml}^{-1}\right)$ or anti-IL10 receptor $\mathrm{Ab}\left(10 \mathrm{ng} \mathrm{ml}^{-1}\right)$. IFN- $\gamma$ and IL-17 production was quantified by enzyme-linked immunosorbent assay $48 \mathrm{~h}$ later. In some cases, MoDCs were sorted from coinfected mice having been treated with anti-IL-10 receptor or isotype control Abs.

Statistical analyses. A Mann-Whitney unpaired $t$-test was used to compare two groups, unless otherwise specified. Comparisons of more than two groups were performed with a nonparametric KruskalWallis one-way analysis of variance, followed by Dunn's post-test (using PRISM software, v.5 GraphPad, San Diego, CA). The survival of infected mice was analyzed using the Kaplan-Meier method and a logrank test. Results are expressed as the mean \pm s.d., unless otherwise stated. The threshold for statistical significance was set to $P<0.05$.

SUPPLEMENTARY MATERIAL is linked to the online version of the paper at http://www.nature.com/mi

\section{ACKNOWLEDGMENTS}

We acknowledge the generous support from the NIAID Tetramer Facility (Emory University, Atlanta, GA) for supplying CD1d tetramers. We gratefully acknowledge Drs T. Nakayama and M Taniguchi (RIKEN Institute, Yokohama, Japan) and $\mathrm{Dr}$ S. Jung (Weizmann Institute of Science, Rehovot, Israel) for the gift of $J \propto 18^{-1-}$ and CD11C-DTR C57BL/6 mice, respectively. Drs P. Gosset (Université Catholique de Lille, Lille, France) and J. Hordeaux are acknowledged for the histological analysis. Drs M. Exley (Manchester University, Manchester, UK) and R. Le gouffic (INRA, Jouy en Jossas, France) are greatly acknowledged for stimulating discussions. This work was supported by the Institut National de la Santé et de la Recherche Médicale (Inserm), the CNRS, the University of Lille, and the Pasteur Institute of Lille. The authors alone are responsible for the content and writing of the paper.

\section{DISCLOSURE}

The authors declared no conflict of interest.

(c) 2017 Society for Mucosal Immunology

\section{REFERENCES}

1. Brundage, J.F. Interactions between influenza and bacterial respiratory pathogens: implications for pandemic preparedness. Lancet Infect. Dis. 6, 303-312 (2006)

2. Morens, D.M., Taubenberger, J.K. \& Fauci, A.S. Predominant role of bacterial pneumonia as a cause of death in pandemic influenza: implications for pandemic influenza preparedness. J. Infect. Dis. 198, 962-970 (2008).

3. Kadioglu, A., Weiser, J.N., Paton, J.C. \& Andrew, P.W. The role of Streptococcus pneumoniae virulence factors in host respiratory colonization and disease. Nat. Rev. Microbiol. 6, 288-301 (2008).

4. McCullers, J.A. Insights into the interaction between influenza virus and pneumococcus. Clin. Microbiol. Rev. 19, 571-582 (2006). 
5. Snelgrove, R.J., Godlee, A. \& Hussell, T. Airway immune homeostasis and implications for influenza-induced inflammation. Trends Immunol. 32, 328-334 (2011).

6. Metzger, D.W. \& Sun, K. Immune dysfunction and bacterial coinfections following influenza. J. Immunol. 191, 2047-2052 (2013).

7. Pittet, L.A., Hall-Stoodley, L., Rutkowski, MR. \& Harmsen, AG. Influenza virus infection decreases tracheal mucociliary velocity and clearance of Streptococcus pneumoniae. Am. J. Respir. Cell. Mol. Biol. 42, 450-460 (2010).

8. Kash, J.C. et al. Lethal synergism of 2009 pandemic H1N1 influenza virus and Streptococcus pneumoniae coinfection is associated with loss of murine lung repair responses. MBio 2, pii: e00172-11 (2011).

9. Ellis, G.T., Davidson, S., Crotta, S., Branzk, N., Papayannopoulos, V. \& Wack, A. TRAlL + monocytes and monocyte-related cells cause lung damage and thereby increase susceptibility to influenza-Streptococcus pneumoniae coinfection. EMBO Rep. 16, 1203-1218 (2015).

10. McNamee, L.A. \& Harmsen, A.G. Both influenza-induced neutrophil dysfunction and neutrophil-independent mechanisms contribute to increased susceptibility to a secondary Streptococcus pneumoniae infection. Infect. Immun. 74, 6707-6721 (2006).

11. Sun, K. \& Metzger, D.W. Inhibition of pulmonary antibacterial defense by interferon-gamma during recovery from influenza infection. Nat. Med. 14, 558-564 (2008).

12. Shahangian, A. et al. Type I IFNs mediate development of postinfluenza bacterial pneumonia in mice. J. Clin. Invest. 119, 1910-1920 (2009).

13. Ghoneim, HE., Thomas, PG. \& McCullers, JA. Depletion of alveolar macrophages during influenza infection facilitates bacterial superinfections. J. Immunol. 191, 1250-1259 (2013).

14. van der Sluijs, K.F. et al. IL-10 is an important mediator of the enhanced susceptibility to pneumococcal pneumonia after influenza infection. J. Immunol. 172, 7603-7609 (2004).

15. Small, C.L. et al. Influenza infection leads to increased susceptibility to subsequent bacterial superinfection by impairing NK cell responses in the lung. J. Immunol. 184, 2048-2056 (2010).

16. Li, W., Moltedo, B. \& Moran, T.M. Type I interferon induction during influenza virus infection increases susceptibility to secondary Streptococcus pneumoniae infection by negative regulation of gammadelta Tcells. J. Virol. 86, 12304-12312 (2012).

17. Cao, J. et al. Activation of IL-27 signalling promotes development of postinfluenza pneumococcal pneumonia. EMBO Mol. Med. 6, 120-140 (2014).

18. Bendelac, A., Savage, P.B. \& Teyton, L. The biology of NKT cells. Annu. Rev. Immunol. 25, 297-336 (2007).

19. Brennan, P.J., Brigl, M. \& Brenner, M.B. Invariant natural killer T cells: an innate activation scheme linked to diverse effector functions. Nat. Rev. Immunol. 13, 101-117 (2013).

20. Paget, C. \& Trottein, F. Role of type 1 natural killer T cells in pulmonary immunity. Mucosal. Immunol. 6, 1054-1067 (2013).

21. Tessmer, M.S., Fatima, A., Paget, C., Trottein, F. \& Brossay, L. NKT cell immune responses to viral infection. Expert Opin. Ther. Targets 13, 153-162 (2009).

22. Ivanov, S., Paget, C. \& Trottein, F. Role of non-conventional T lymphocytes in respiratory Infections: the case of the Pneumococcus. PLoS Pathog. 10 , e1004300 (2014).

23. Kawakami, K. et al. Critical role of Valpha14 + natural killer T cells in the innate phase of host protection against Streptococcus pneumoniae infection. Eur. J. Immunol. 33, 3322-3330 (2003).

24. Nakamatsu, M. et al. Role of interferon-gamma in Valpha14 + natural killer Tcell-mediated host defense against Streptococcus pneumoniae infection in murine lungs. Microbes Infect. 9, 364-374 (2007).

25. Brigl, M. et al. Innate and cytokine-driven signals, rather than microbial antigens, dominate in natural killer $\mathrm{T}$ cell activation during microbial infection. J. Exp. Med. 208, 1163-1177 (2011).

26. Kinjo, $Y$. et al. Invariant natural killer T cells recognize glycolipids from pathogenic Gram-positive bacteria. Nat. Immunol. 12, 966-974 (2011).

27. Lisbonne, M. et al. Cutting edge: invariant $V$ alpha 14 NKT cells are required for allergen-induced airway inflammation and hyperreactivity in an experimental asthma model. J. Immunol. 171, 1637-1641 (2003).

28. Pichavant, M. et al. Oxidative stress-mediated iNKT-cell activation is involved in COPD pathogenesis. Mucosal Immunol. 7, 568-578 (2014).
29. De Santo, C. et al. Invariant NKT cells reduce the immunosuppressive activity of influenza A virus-induced myeloid-derived suppressor cells in mice and humans. J. Clin. Invest. 118, 4036-4048 (2008).

30. Paget, C. et al. Potential role of invariant NKT cells in the control of pulmonary inflammation and CD8 + Tcell response during acute influenza A virus H3N2 pneumonia. J. Immunol. 186, 5590-5602 (2011).

31. Paget, C. et al. Interleukin-22 is produced by invariant natural killer T lymphocytes during influenza $A$ virus infection: potential role in protection against lung epithelial damages. J. Biol. Chem. 287, 8816-8829 (2012).

32. Kok, W.L. et al. Pivotal advance: invariant NKT cells reduce accumulation of inflammatory monocytes in the lungs and decrease immune-pathology during severe influenza A virus infection. J. Leukoc. Biol. 91, 357-368 (2012).

33. Ivanov, S. etal. Interleukin-22 reduces lung inflammation during influenza A virus infection and protects against secondary bacterial infection. J. Virol. 87, 6911-6924 (2013).

34. Gorski, S.A., Hahn, Y.S. \& Braciale, T.J. Group 2 innate lymphoid cell production of IL-5 is regulated by NKT cells during influenza virus infection. PLoS Pathog. 9, e1003615 (2013).

35. Monticelli, L.A., Sonnenberg, G.F., Abt, M.C., Alenghat, T., Ziegler, C.G. \& Doering, TA. et al. Innate lymphoid cells promote lung-tissue homeostasis after infection with influenza virus. Nat. Immunol. 12, 1045-1054 (2011).

36. Venken, K., Decruy, T., Aspeslagh, S., Van Calenbergh, S., Lambrecht, B.N. \& Elewaut, D. Bacterial CD1d-restricted glycolipids induce IL-10 production by human regulatory T cells upon cross-talk with invariant NKT cells. J. Immunol. 191, 2174-2183 (2013).

37. Kumar, P., Thakar, M.S., Ouyang, W. \& Malarkannan, S. IL-22 from conventional NK cells is epithelial regenerative and inflammation protective during influenza infection. Mucosal Immunol. 6, 69-82 (2013).

38. Pociask, D.A. et al. IL-22 is essential for lung epithelial repair following influenza infection. Am. J. Pathol. 182, 1286-1296 (2013).

39. Didierlaurent, A. et al. Sustained desensitization to bacterial Toll-like receptor ligands after resolution of respiratory influenza infection. J. Exp. Med. 205, 323-329 (2008).

40. Sun, J., Madan, R., Karp, C.L. \& Braciale, T.J. Effector T cells control lung inflammation during acute influenza virus infection by producing IL-10. Nat. Med. 15, 277-284 (2009).

41. Bedoya, F. et al. Viral antigen induces differentiation of Foxp3 + natural regulatory $T$ cells in influenza virus-infected mice. J. Immunol. 190, 6115-6125 (2013).

42. Yamaura, A., Hotta, C., Nakazawa, M., Van Kaer, L. \& Minami, M. Human invariant Valpha24 + natural killer $T$ cells acquire regulatory functions by interacting with IL-10-treated dendritic cells. Blood 111, 4254-4263 (2008).

43. McGill, J., Van Rooijen, N. \& Legge, K.L. IL-15 trans-presentation by pulmonary dendritic cells promotes effector CD8 T cell survival during influenza virus infection. J. Exp. Med. 207, 521-534 (2010).

44. Blevins, L.K. et al. Coinfection with Streptococcus pneumoniae negatively modulates the size and composition of the ongoing influenza-specific CD8(+) T cell response. J. Immunol. 193, 5076-5087 (2014).

45. Kudva, A. et al. Influenza A inhibits Th17-mediated host defense against bacterial pneumonia in mice. J. Immunol. 186, 1666-1674 (2011).

46. Robinson, K.M. et al. Influenza A exacerbates Staphylococcus aureus pneumonia by attenuating IL-1 beta production in mice. J. Immunol. 191, 5153-5159 (2013).

47. van der Sluijs, K.F. et al. Influenza-induced expression of indoleamine 2,3-dioxygenase enhances interleukin-10 production and bacterial outgrowth during secondary pneumococcal pneumonia. J. Infect. Dis. 193, 214-222 (2006).

48. Cui, J. et al. Requirement for Valpha14 NKT cells in IL-12-mediated rejection of tumors. Science 278, 1623-1626 (1997).

49. Jung, S. et al. In vivo depletion of CD11c + dendritic cells abrogates priming of CD $8+$ Tcells by exogenous cell-associated antigens. Immunity 17, 211-220 (2002).

50. Bouabe, H., Liu, Y., Moser, M., Bosl, M.R. \& Heesemann, J. Novel highly sensitive IL-10-beta-lactamase reporter mouse reveals cells of the innate immune system as a substantial source of IL-10 in vivo. J. Immunol. 187, 3165-3176 (2011). 